\title{
Paper Ku Band Bethe Hole Coupler Using Gap Waveguide Technology
}

\author{
Efat Nematpour, Mohamad Hossein Ostovarzadeh, and Seyed Ali Razavi \\ Graduate University of Advanced Technology, Kerman, Iran
}

https://doi.org/10.26636/jtit.2019.132119

\begin{abstract}
The gap waveguide technology is a new technique used for designing and fabricating microwave components, ensuring a low-loss and easy fabrication process, especially at high frequencies, and allowing for the production of multilayer structures due to the lack of requirement of an electrical connection between the metal layers of the waveguide structure. This paper presents the design and areas of implementation of single-hole and multi-hole $20 \mathrm{~dB}$ Bethe couplers, using the groove gap waveguide (GGW) technology for Ku band. Simulation results show that the operating bandwidth of the proposed design is over $40 \%$ wider, and its isolation rate is more than $25 \mathrm{~dB}$ higher. By using the multi hole configuration, a bandwidth that is more than $59 \%$ wider and the isolation rate of over $30 \mathrm{~dB}$ may be obtained.
\end{abstract}

Keywords-Bethe hole coupler, multi-hole coupler, groove gap waveguide.

\section{Introduction}

Microwave components are currently designed using conventional metallic waveguides, microstrip lines, strip lines and SIW. Such technologies suffer from certain disadvantages that limit their application. Conventional waveguides offer the lowest loss rates and the highest power handling capabilities. However, the process of their fabrication is expensive challenging at high frequencies, due to the need to provide a fine electrical contact between metal plates. Microstrip and coplanar lines are a low-cost solution that is suitable for integrating active microwave components on a PCB, but they suffer from a high insertion loss rate at high-frequencies, and offer a low power handling capability. SIW structures are compact, planar and generate no undesired radiation, but they suffer from high dielectric and radiation losses at millimeter wave frequency ranges, due to the discrete nature of sidewalls which do not provide a perfect shielding [1]-[3].

The gap waveguide technology has recently gained in popularity due to its easy manufacturing combined with the lack of the requirement to ensure an electric contact between the metal plates, especially at millimeter-wave frequencies. The other advantages of the solution include the following: easy assembly (especially for multi-layer configurations) and low loss rates due to the lack of presence of a dielectric material in the propagation path, as well as no parasitic radiation and high-quality rates. So far, several microwave components based on the gap waveguide technology have been implemented, including various types of antennas [4]-[9], power combiners [10], diplexerpower dividers [11] and filters [12], [13]. Four different types of gap waveguide structures include the following: ridge gap waveguide (RGW) [13], groove gap waveguide (GGW) [14], inverted microstrip gap waveguide [15] and microstrip-ridge gap waveguide [16].

The purpose of this work is to design a Bethe hole coupler using the groove gap waveguide technology. In Section 2, we discuss the GGW structure. The design and simulation results of single-hole and multi-hole Bethe couplers are presented in Sections 3 and 4, respectively. In Section 5, the performance of both couplers is compared. Finally, in Section 6, concluding remarks are presented.

\section{Groove Gap Waveguide Structure}

GGW technology is based on the propagation of waves in the gap between two parallel metallic surfaces providing a non-metal contact-guiding structure [14]. Depending on the geometry of the groove, it is possible to use vertical (GGW-VP) or horizontal (GGW-HP) polarizations [17], (a)

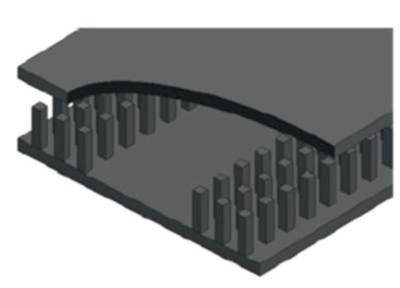

(c)

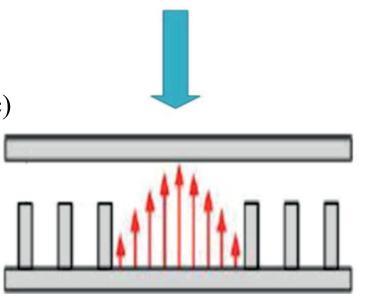

(b)

(d)
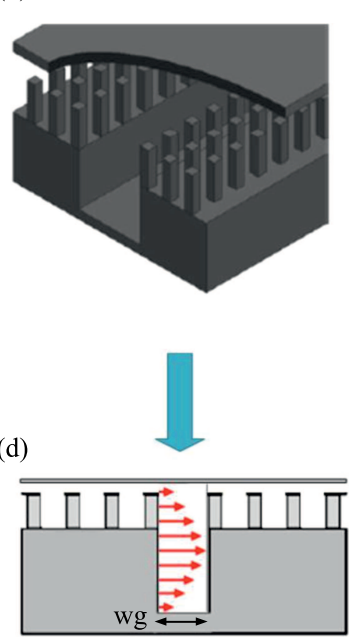

Fig. 1. GGW geometry: (a) GGW-VP, (b) GGW-HP, (c) E field distribution in GGW-VP, (d) E field distribution in GGW-HP. 
as shown in Fig. 1. GGW is equivalent to a conventional rectangular waveguide that supports TE modes. There is no need to ensure electric contact between the top and bottom plates of the waveguide, which makes it suitable for designing microwave circuits operating at high frequencies. In this paper the GGW-VP version of the design is examined, based on the concept and design guidelines originating from [14].

\section{Single-hole Bethe Coupler Design}

The geometry of the proposed GGW Bethe hole coupler is shown in Fig. 2. It includes two layers of GGW which are coupled together through one circular hole located in the broad wall. $3 \mathrm{D}$ and $2 \mathrm{D}$ views of the structure are depicted in Fig. 2a and in Fig. 2b-c, respectively. The initial $r_{0}$ value for the hole diameter and its position relative to the center line of GGW are calculated using the formulas from [18] for the coupling coefficient of $-20 \mathrm{~dB}$. According to [19], zero thickness is considered for the coupling slot. Then, fine tuning is performed by full wave simulation of HFSS software to obtain optimum performance in terms of bandwidth, for the case in which the coupling slot has non-zero thickness.

The impact of slot thickness on the coupling coefficient is investigated for three frequencies within the operating

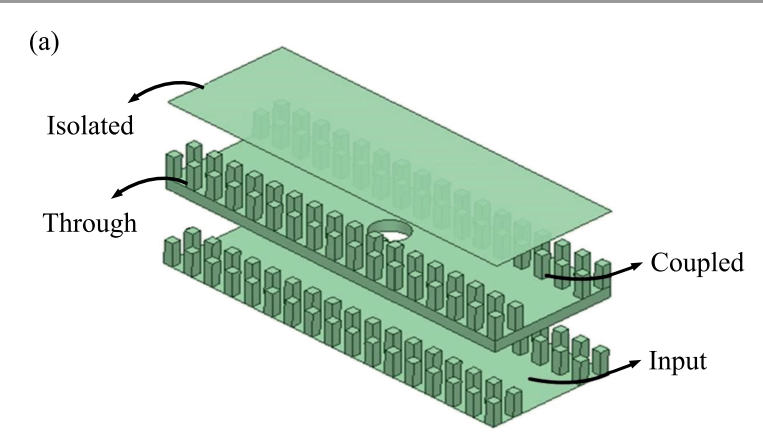

(b)
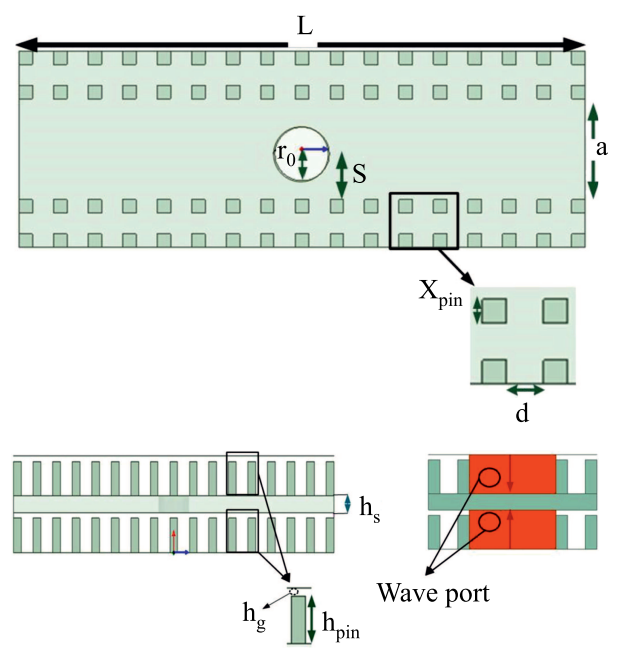

Fig. 2. GGW single-slot Beth hole coupler structure: (a) the expanded 3D view, (b) top view, (c) side views.

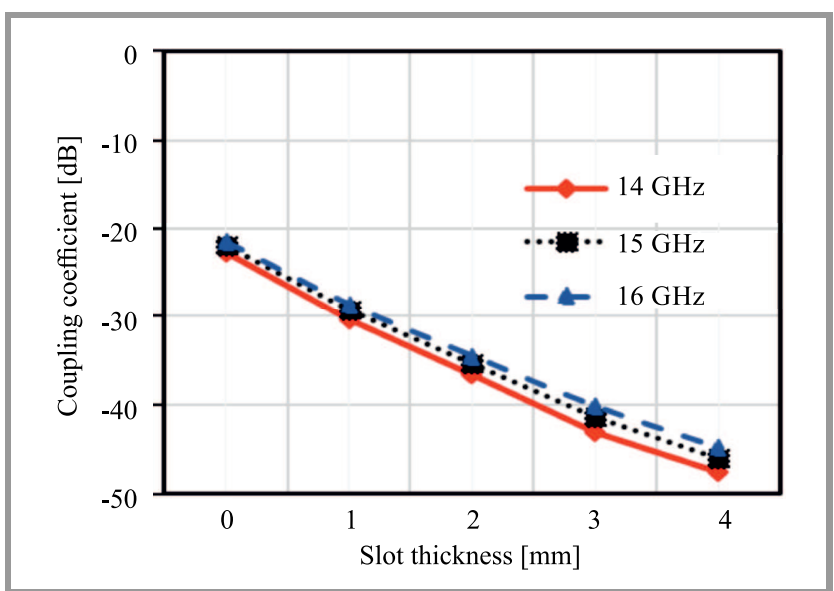

Fig. 3. The impact of slot thickness on the coupling coefficient $\left(r_{0}=2.7 \mathrm{~mm}\right)$.

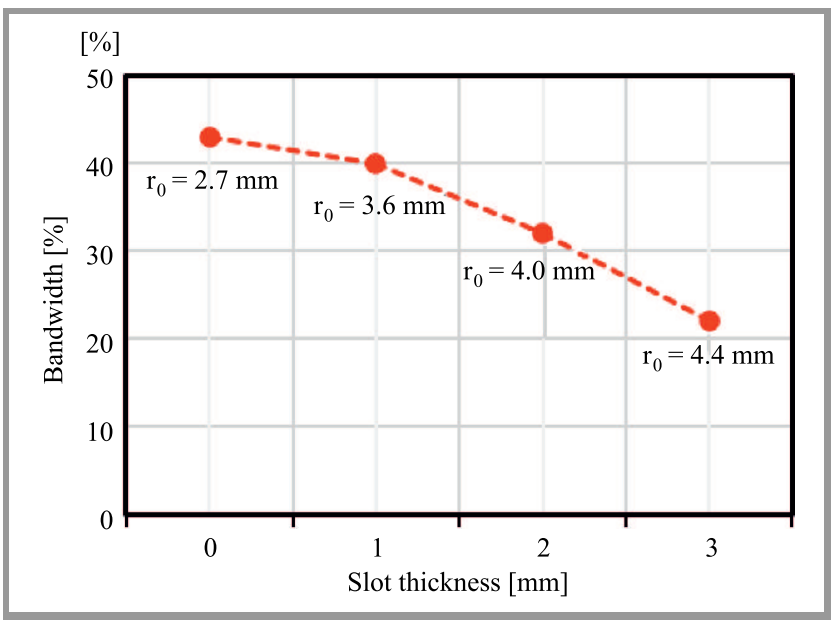

Fig. 4. The impact of slot thickness on bandwidth for the fixed coupling coefficient of $-20 \mathrm{~dB}$.

Table 1

Parameters of the designed coupler

\begin{tabular}{|c|c|}
\hline Parameter & Value $[\mathrm{mm}]$ \\
\hline \hline$a$ & 15 \\
\hline$d$ & 3.1 \\
\hline$h_{\mathrm{g}}$ & 1 \\
\hline$h_{\text {pin }}$ & 6 \\
\hline$h_{\mathrm{s}}$ & 1 \\
\hline$L$ & 83.6 \\
\hline$r_{0}$ & 3.6 \\
\hline$S$ & 6.8 \\
\hline$x_{\text {pin }}$ & 2 \\
\hline
\end{tabular}

bandwidth, as shown in Fig. 3. It can be observed that the coupling coefficient decreases as slot thickness increases. To compensate for this effect, the slot radius can be increased in order to enhance the coupling coefficient. The maximum bandwidth for the coupling coefficient $-20 \mathrm{~dB}$ could be obtained using different slot thicknesses and cor- 
responding slot radiuses, as shown in Fig. 4. For a fixed coupling coefficient $(-20 \mathrm{~dB})$, as the slot thickness increases, a larger coupling slot is needed. However, the operating bandwidth decreases due to the increasing difficulty in matching impedance between upper and lower layers. Based on the results shown in Figs. 3 and 4, the coupling slot thickness of $1 \mathrm{~mm}$ was selected to ensure maximum bandwidth. The calculated dimensions of the designed $20 \mathrm{~dB}$ coupler are listed in Table 1.

The coupler design was then simulated using HFSS software, and the results are compared with those obtained means of CST software.

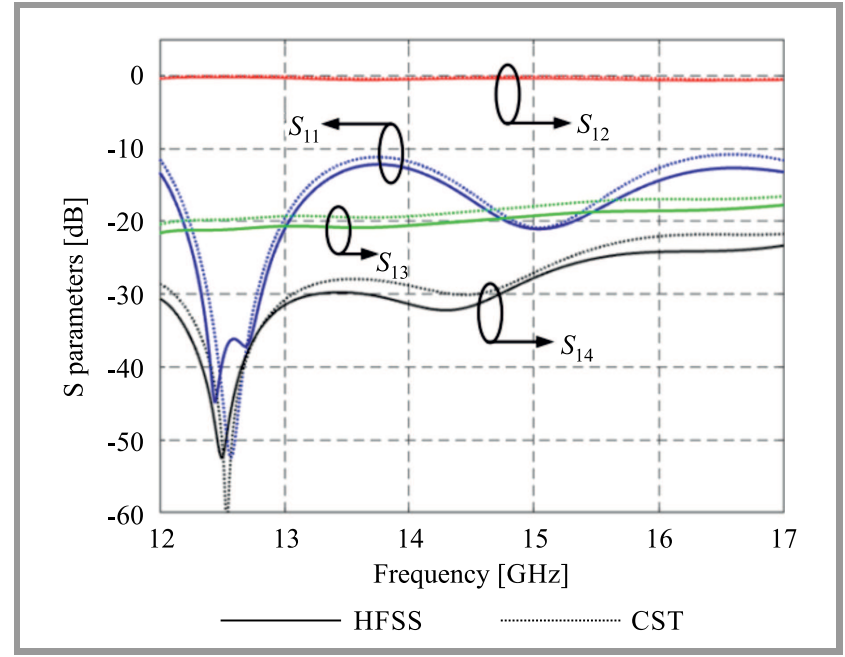

Fig. 5. $S$ parameters of the designed coupler.

The simulation results of the designed single-hole Bethe coupler are shown in Fig. 5. $S_{11}$ is below $-10 \mathrm{~dB}$ over the entire bandwidth $(12-17 \mathrm{GHz})$, while $S_{12}$ is above $-0.5 \mathrm{~dB}$. The coupling coefficient $C$ is about $-20 \pm 2 \mathrm{~dB}$, and the isolation rate is better than $-25 \mathrm{~dB}$ over the entire bandwidth. Table 2 summarizes the results.

Table 2

$S$ parameters and corresponding bandwidth

\begin{tabular}{|c|c|}
\hline$S$ parameters boundary & Frequency $[\mathrm{GHz}]$ \\
\hline \hline BW $\left(S_{11}<-10 \mathrm{~dB}\right)$ & $12-18(50 \%)$ \\
BW $(C=-20 \pm 2 \mathrm{~dB})$ & $12-16.82(40.16 \%)$ \\
$S_{14}<-25 \mathrm{~dB}$ & $12-17$ \\
$S_{12}>-0.5$ & $12-18$ \\
\hline
\end{tabular}

\section{Multi-hole Coupler Design}

The multi-hole technique is used to increase the directivity and bandwidth. To eliminate output power at port 4 , the distances between the holes must be about $\lambda_{g} / 4$, where $\lambda_{g}$ is the guiding wavelength of the dominant $\mathrm{TE}_{10}$ mode at the center frequency [18]. In Fig. 6, the geometry of the designed four-hole Chebyshev coupler for $\mathrm{Ku}$ band is shown. Formulas from [18] were used to calculate the dimensions of the multi-hole coupler for the coupling coefficient of $-20 \mathrm{~dB}$, with the thickness of coupling slots being considered to equal zero. 3D and $2 \mathrm{D}$ views of the coupler design are shown in Fig. 6a and Fig. 6b, respectively.

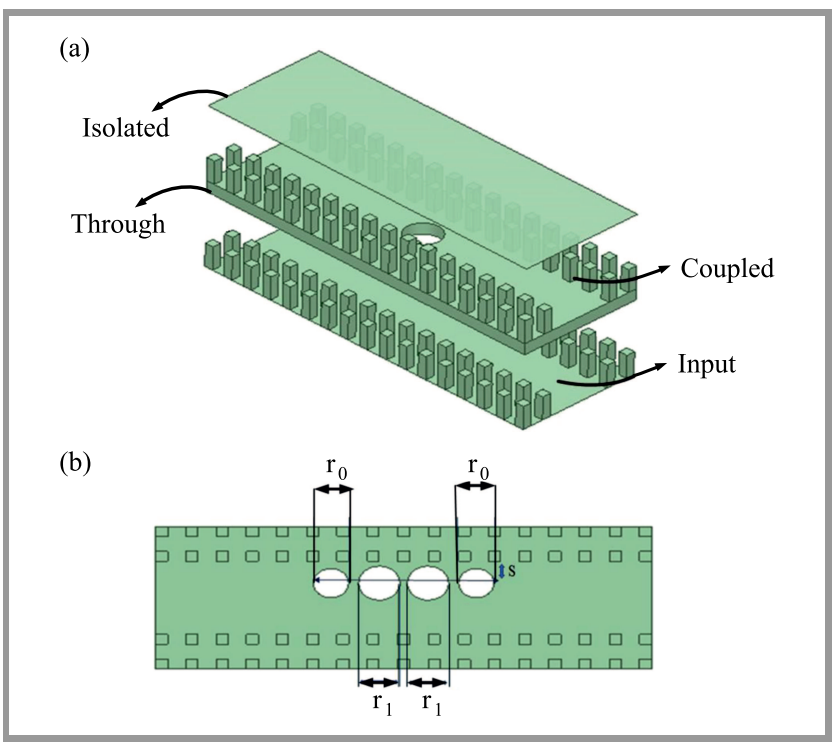

Fig. 6. Multi-hole GGW Bethe hole coupler: (a) 3D view, (b) top view.

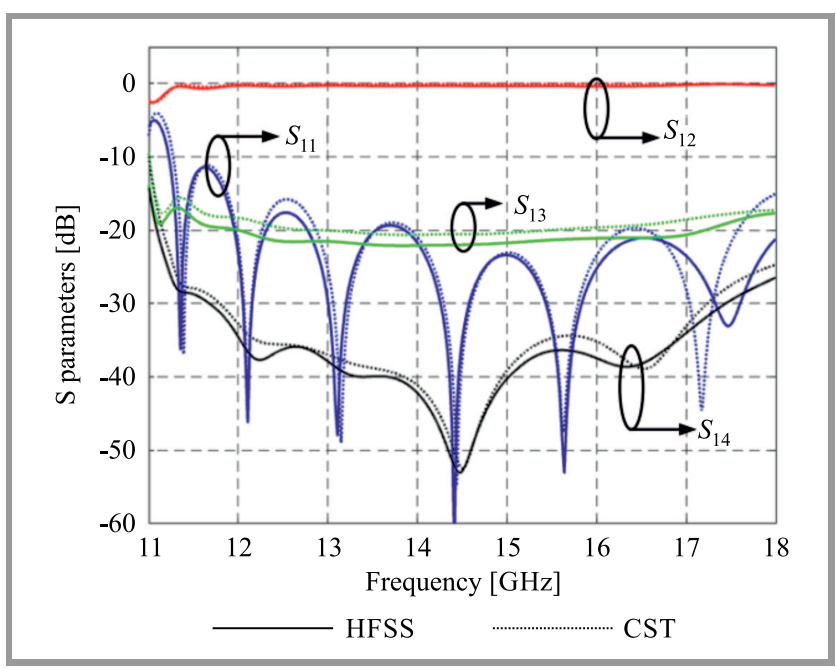

Fig. 7. $S$ parameters of the designed $20 \mathrm{~dB}$ multi-hole coupler.

Similarly to the single-hole coupler, the effect that the thickness of slots has on the coupling coefficient are investigated at three frequencies within the operating bandwidth, and the same behavior as in the case of the single-hole coupler was observed. To achieve the desired coupling coefficient of $-20 \mathrm{~dB}$, the dimensions of the slots are fine-tuned: $r_{0}=2.8 \mathrm{~mm}, r_{1}=3.1 \mathrm{~mm}$, and $S=3.75 \mathrm{~mm}$.

The $S$ parameters for the coupling slot thickness of 1-mm are shown in Fig. 7 and in Table 3.

$S_{11}$ is lower than $-10 \mathrm{~dB}$ and $S_{12}$ is more than $-0.3 \mathrm{~dB}$ over the majority of the frequency band, evidencing proper impedance matching, as well as a low loss rate. The coupling coefficient $S_{13}$ is almost $-20 \mathrm{~dB}$, which indicates 
Table 3

Summary of simulation results

\begin{tabular}{|c|c|}
\hline$S$ parameters boundary & Frequency $[\mathrm{GHz}]$ \\
\hline \hline BW $\left(S_{11}<-10 \mathrm{~dB}\right)$ & $11-20(81.81 \%)$ \\
BW $(C=-20 \pm 2 \mathrm{~dB})$ & $11.22-17.86(59.44 \%)$ \\
$S_{14}<-30 \mathrm{~dB}$ & $11-17.51$ \\
$S_{12}>-0.3$ & $11-20$ \\
\hline
\end{tabular}

a flat and proper coupling performance. $S_{14}$ also remains below $-30 \mathrm{~dB}$, which confirms very good isolation between ports.

\section{Comparison of Single-hole and Multi-hole Couplers}

The results shown in Figs. 5 and 7 confirm the improvement in the structure's performance in terms of insertion loss $\left(S_{12}\right)$, return loss $\left(S_{11}\right)$, bandwidth and isolation, with the said improvement originating from the increasing number of holes. In both cases the coupling coefficient is very near to the desired value of $-20 \mathrm{~dB}$, and it remains flat over the entire bandwidth. Figure 8 compares the performance of the designed single- and multi-hole couplers in terms of directivity, showing that the multi-hole version provides higher directivity.

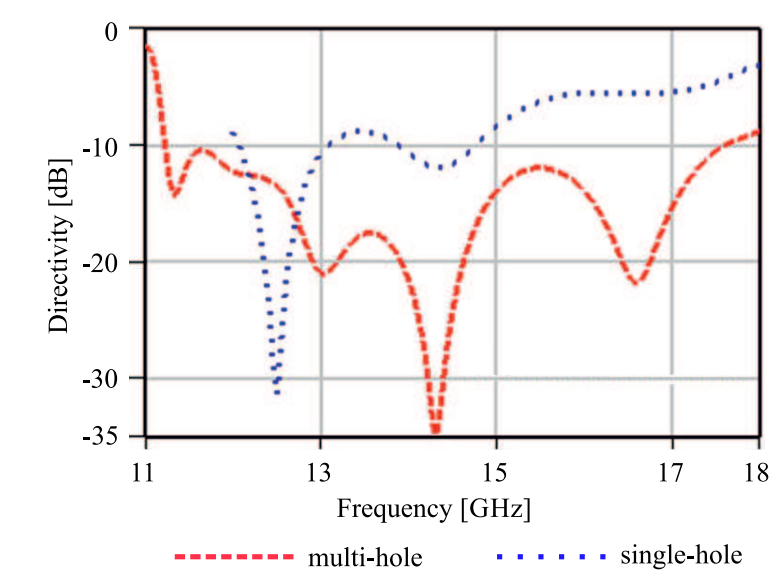

Fig. 8. Directivity of both structures.

\section{Conclusions}

In this paper, two types of $20 \mathrm{~dB}$ Bethe couplers are designed and simulated using groove gap waveguide technology. Simulation results show desirable performance of both designed structures. The couplers are suitable for $\mathrm{Ku}$ band operation. However, it is observed that the multi-hole version shows better performance in terms of insertion loss, return loss, bandwidth and directivity.

\section{References}

[1] M. Bozzi, M. Pasian, L. Perregrini, and K. Wu, "On the losses in substrate integrated waveguides", in Proc. Eur. Microwave Conf., Munich, Germany, 2007, pp. 384-387

(doi: 10.1109/EUMC.2007.4405207).

[2] F. Xu and K. Wu, "Guided-wave and leakage characteristics of substrate integrated waveguide", IEEE Trans. on Microw. Theory and Techniq., vol. 53, no. 1, pp. 66-72, 2005

(doi: 10.1109/TMTT.2004.839303).

[3] M. Pasian, M. Bozzi, and L. Perregrini, "A formula for radiation loss in substrate integrated waveguide", IEEE Trans. on Microwave Theory and Techniques, vol. 62, no. 10, 2014

(doi: 10.1109/TMTT.2014.2341663).

[4] J. Liu, A. U. Zaman, and J. Yang, "Design of wideband slot array antenna by groove gap waveguide in millimeter waves", in Proc. IEEE-APS Topical Conf. on Antenn. and Propag. in Wirel. Commun. APWC 2018, Cartagena des Indias, Colombia, 2018, pp. 725-728 (doi: 10.1109/APWC.2018.8503800).

[5] A. Vosoogh et al., " $W$-band low-profile monopulse slot array antenna based on gap waveguide corporate-feed network", IEEE Trans. on Antenn. and Propag., vol. 66, no. 12, pp. 6997-7009, 2018 (doi: 10.1109/TAP.2018.2874427).

[6] L. Wang et al., "Low-dispersive leaky-wave antenna integrated in groove gap waveguide technology", IEEE Trans. on Antenn. and Propag., vol. 66, no. 11, pp. 5727-5736, 2018 (doi: 10.1109/TAP.2018.2863115).

[7] D. Zarifi, A. Farahbakhsh, A. U. Zaman, and P.-S. Kildal, "Design and fabrication of a high-gain $60-\mathrm{GHz}$ corrugated slot antenna array with ridge gap waveguide distribution layer, IEEE Trans. on Antenn. and Propag., vol. 64, no. 7, pp. 2905-2913, 2016 (doi: 10.1109/TAP.2016.2565682).

[8] A. U. Zaman and P.-S. Kildal, "Different gap waveguide slot array configurations for mmwave fixed beam antenna application", in Proc. 10th Eur. Conf. on Antenn. and Propag. EuCAP 2016, Davos, Switzerland, 2016, pp. 1-4 (doi: 10.1109/EuCAP.2016.7481541).

[9] A. Vosoogh et al., "E-band 3-D metal printed wideband planar horn array antenna”, in Proc. In. Symp. on Antenn. and Propag. ISAP2016, Okinawa, Japan, 2016, pp. 304-305.

[10] R. Maaskant et al., "Spatial power combining and splitting in gap waveguide technology", IEEE Microw. and Wirel. Components Lett., vol. 26, no. 7, pp. 472-474, 2016 (doi: 10.1109/LMWC.2016.2574828).

[11] M. S. Sorkherizi, A. Vosoogh, A. A. Kishk, and P.-S. Kildal, "Design of integrated diplexer-power divider", in Proc. IEEE MTT-S Int. Microw. Symp. IMS 2016, San Francisco, CA, USA, 2016 (doi: 10.1109/MWSYM.2016.7540124).

[12] A. Vosoogh, A. A. Brazález, and P.-S. Kildal, "A V-band inverted microstrip gap waveguide end-coupled bandpass filter", IEEE $\mathrm{Mi}$ crow. and Wirel. Compon. Lett., vol. 26, no. 4, pp. 261-263, 2016 (doi: 10.1109/LMWC.2016.2538598).

[13] P.-S. Kildal et al., "Design and experimental verification of ridge gap waveguide in bed of nails for parallel-plate mode suppression", IET Microw., Antenn. and Propag., vol. 5, no. 3, pp. 262-270, 2011 (doi: 10.1049/iet-map.2010.0089).

[14] E. Rajo-Iglesias and P.-S. Kildal, "Groove gap waveguide: A rectangular waveguide between contactless metal plates enabled by parallel-plate cut-off", in Proc. of the 4th Eur. Conf. on Antenn. and Propag., Barcelona, Spain, 2010.

[15] A. A. Brazalez, A. U. Zaman, and P.-S. Kildal, "Improved microstrip filters using PMC packaging by lid of nails", IEEE Trans. on Compon., Packag. and Manufact. Technol., vol. 2, no. 7, pp. 1075-1084, 2012 (doi: 10.1109/TCPMT.2012.2190931).

[16] A. Valero-Nogueira et al., "Gap waveguides using a suspended strip on a bed of nails", IEEE Antenn. and Wirel. Propag. Lett., vol. 10, pp. 1006-1009, 2011 (doi: 10.1109/LAWP.2011.2167591).

[17] L. F. Carrera-Suárez et al., "A novel twist between Gap Waveguides for compact slot-array antennas", in Proc. IEEE Antenn. and Propag. Soc. Int. Symp. APSURSI 2014, Memphis, TN, USA, 2014, pp. 456-457 (doi: 10.1109/APS.2014.6904559). 
[18] D. M. Pozar, Microwave Engineering, 4th ed. Wiley, 2011 (ISBN: 978-0-470-63155-3).

[19] H. Bethe, "Theory of diffraction by small holes", Phys. Rev., vol. 66, pp. 163-182, 1944 (doi: 10.1103/PhysRev.66.163).

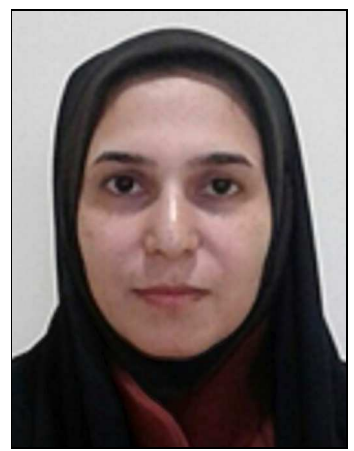

Efat Nematpour received her B.Sc. degree in Electrical Engineering from the University of Lorestan, Iran. She works at the Department of Electrical and Computer Engineering, Graduate University of Advanced Technology, Kerman, Iran.

E-mail: Efatnematpour@yahoo.com Graduate University of Advanced Technology Kerman, Iran

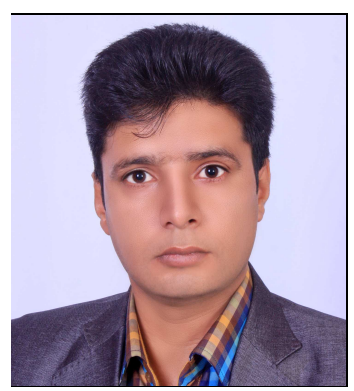

Mohamad Hossein Ostovarzadeh received his B.Sc. degree in Electrical Engineering from Shahid Bahonar University, Kerman, Iran, in 2004, and M.Sc. degree in Telecommunications from the Sharif University of Technology, Teheran, Iran, in 2006. He received his
Ph.D. degree in Electrical Engineering from the Amirkabir University of Technology. His research interests are in the field of electromagnetic non-destructive testing techniques and microwave circuits.

E-mail: mh.ostovarzadeh@kgut.ac.ir

Graduate University of Advanced Technology

Kerman, Iran

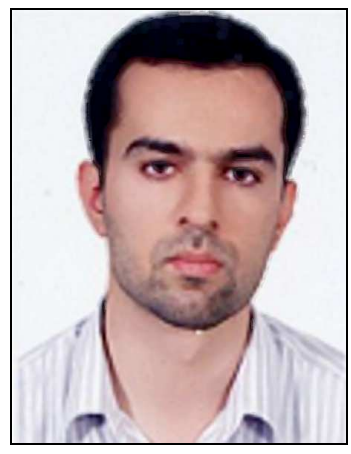

Seyed Ali Razavi received his B.Sc. degree in Electrical Engineering from the University of Shahid Bahonar, Kerman, Iran, in 2006 and M.Sc. in Electrical Engineering from Ferdowsi University of Mashhad, Iran, in 2009. He received his Ph.D. degree in Electrical Engineering from Ferdowsi University of Mashhad, Iran, in 2013. Currently, he works at the Graduate University of Advanced Technology, Kerman, Iran, as an Assistant Professor. He also collaborates with the group of antenna experts at the Chalmers University of Technology. His interests focus on microwave and millimeter-wave passive devices, antennas, electromagnetic wave scattering, SIW and gap-waveguide technologies.

E-mail: s.razaviparizi@kgut.ac.ir

Graduate University of Advanced Technology

Kerman, Iran 